\title{
Histotripsy of VX-2 Tumor Implanted in a Renal Rabbit Model
}

\author{
Nicholas R. Styn, M.D., Jeffery C. Wheat, M.D., \\ Timothy L. Hall, Ph.D., ${ }^{2}$ and William W. Roberts, M.D., ${ }^{1,2}$
}

\begin{abstract}
Introduction: Histotripsy is an extracorporeal focused ultrasound (US) technology that uses controlled cavitation to induce nonthermal mechanical tissue fractionation. Feasibility of histotripsy ablation of normal renal tissue in an in vivo rabbit model has previously been demonstrated. Our specific objective in this study was to characterize the histologic effects of histotripsy on VX-2 tumor implanted in the kidneys of an in vivo rabbit model. Methods: VX-2 tumor was implanted below the renal capsule in $15 \mathrm{New}$ Zealand white rabbits. Two weeks after implantation, tumors were localized with diagnostic US imaging. Targeted volumes within the observed tumor were treated with short $(3 \mu \mathrm{s})$ pulses of $1 \mathrm{MHz}$ acoustic energy at a repetition frequency of $300 \mathrm{~Hz}$. Twenty tumors were treated with histotripsy and 7 served as tumor controls. Three normal kidneys were also treated with histotripsy. Kidneys and lungs were harvested, grossly inspected, and processed for histopathologic analysis.

Results: Real-time US imaging confirmed presence of cavitation during all histotripsy treatments. Examination of tumor and kidney specimens revealed $100 \%$ tumor growth with an average tumor diameter of $7 \mathrm{~mm}$ (range 212). In 16 of 20 tumors treated with histotripsy, acellular zones of debris and finely disrupted cellular architecture were present on histology. Kidneys harvested 24 hours after treatment revealed an extensive inflammatory reaction.

Conclusions: Transcutaneous application of histotripsy to implanted VX-2 tumor in rabbit kidney produced fractionation of malignant tissue. These findings support the further study and development of histotripsy for potential oncologic application.
\end{abstract}

\section{Introduction}

$\mathbf{H}$ ISTOTRIPSY Is A noninvasive focused ultrasound (US) technology that delivers short bursts $(1-10 \mu \mathrm{s})$ of acoustic energy to a targeted volume at a low $(<1 \%)$ duty cycle to induce cavitation with negligible thermal contribution. ${ }^{1} \mathrm{Ca}-$ vitation is a phenomenon where rapid cycling from compressive to rarefactive pressures generates a highly dynamic cluster of microbubbles that oscillate and collapse producing mechanical tissue fractionation. ${ }^{1-4}$ Histologic analysis of tissues after application of histotripsy typically demonstrates architectural disruption and a slurry of subcellular debris. ${ }^{5}$ Histotripsy is different from high-intensity focused US and other conventional ablative modalities as the destructive force is mechanical, not thermal. ${ }^{6-8}$

The effect of histotripsy treatment on normal kidney tissue has previously been studied. A wide range of pulse repetition frequencies and peak acoustic intensities were evaluated in an in vitro porcine kidney model to identify acoustic parameter regions with distinct modes of ablation (nonthermal, thermal, and mixed). ${ }^{8}$ The parameter region yielding nonthermal damage characterized by cell fractionation and tissue architecture disruption without evidence of thermal coagulative necrosis was defined to be the histotripsy region. Histotripsy was applied in an in vivo rabbit model establishing the feasibility of extracorporeal tissue fractionation. ${ }^{1}$ A chronic in vivo study was then performed to assess local tissue response to histotripsy. ${ }^{5}$ In the first week after histotripsy treatment, an acute inflammatory response was seen within and around the targeted regions consistent with a typical wound healing response. Resorption of the fractionated material occurred rapidly and was completed by 60 days with minimal scar formation. ${ }^{5}$ These exciting preliminary results have provided the motivation for continued research and development of histotripsy as a potential noninvasive treatment modality for small renal masses and other solid organ malignancies.

$\mathrm{VX}-2$ is a rabbit papilloma cell line that has been used as a model for malignancy in several organs, including liver,

Departments of ${ }^{1}$ Urology and ${ }^{2}$ Biomedical Engineering, University of Michigan, Ann Arbor, Michigan. 
rectum, and kidney. ${ }^{9-13}$ Injection of VX-2 suspension and direct implantation of tumor into the rabbit kidney have both been described with good tumor growth. Our objective in this study was to characterize the histologic effects after histotripsy treatment of VX-2 tumor implanted in renal tissue and thereby demonstrate feasibility of histotripsy tumor ablation.

\section{Methods}

A total of 27 kidneys were implanted with VX-2 tumor. Twenty were treated with histotripsy and 7 served as controls. Three nonimplanted kidneys were also treated to validate previous effects of histotripsy on normal renal parenchyma. All described procedures and protocols were approved by the University of Michigan Committee for Use and Care of Laboratory Animals.

Viable VX-2 tumor tissue was harvested from the hind-legs of carrier rabbits immediately after euthanasia, sectioned into $2 \times 2 \times 2 \mathrm{~mm}$ cubes, and maintained in Hanks balanced solution (Krackeler Scientific Inc.) for implantation within 3 hours. To minimize variability of tumor behavior during the course of this study, viable VX-2 cells were continuously maintained in a series of carrier rabbits.

\section{Tumor implantation}

Fifteen adult New Zealand White rabbits weighing 2.5 to $3.5 \mathrm{~kg}$ were anesthetized using intramuscular injections of xylazine $5 \mathrm{mg} / \mathrm{kg}$ and ketamine $35 \mathrm{mg} / \mathrm{kg}$. Fur from the flank regions bilaterally was removed with electric clippers. After endotracheal intubation, anesthesia was maintained with forced ventilation of $1 \%$ to $2 \%$ isoflurane. Vital signs (heart rate, oxygen saturation, respiratory rate, and temperature) were monitored. All rabbits were given Penicillin-G Benzathine $(40,000 \mathrm{IU} / \mathrm{kg})$ before laparotomy.

A 4-cm laparotomy was made $1 \mathrm{~cm}$ inferior to the costal margin and carried into the peritoneal space. The kidney was identified and pararenal fat cleared from the dorsal surface of the kidney. A $2 \times 2 \times 2 \mathrm{~mm}$ piece of VX-2 tumor was inserted under the renal capsule in the lateral aspect of the central region of the kidney. A surgical clip was placed adjacent to the tumor as a fiducial marker. The dorsal surface of the kidney was sutured to the abdominal wall musculature. Twelve rabbits underwent bilateral tumor implantation and three rabbit underwent unilateral implantation.

\section{Experimental setup and procedure}

The histotripsy therapeutic US system (Fig. 1) consisted of four confocally aligned piezocomposite therapy transducers (Imasonic) and a $10 \mathrm{MHz}$ linear imaging probe (General Electric). Each transducer was square in shape with a $50 \mathrm{~mm}$ width and $70 \mathrm{~mm}$ focal distance and operated at $1 \mathrm{MHz}$. The transducer system was placed within a tank of warm $\left(38^{\circ} \mathrm{C}\right)$ degassed water.

Two weeks after implantation, the rabbits were anesthetized and fur was again removed from the flank region with clippers. Kidneys were imaged with a $10 \mathrm{MHz}$ US imaging transducer to localize the tumor. Each rabbit was then placed in decubitus position on a thin plastic membrane suspended in the tank containing the therapeutic transducer system. The three-axis positioning system allowed precise translation of the rabbit and localization of the tumor within the

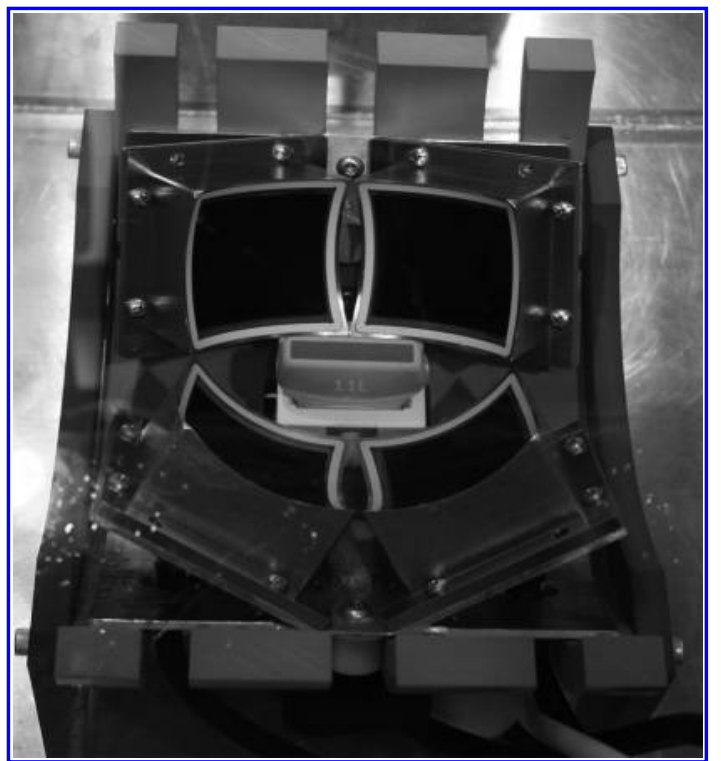

FIG. 1. Histotripsy therapeutic transducer composed of four individual $1-\mathrm{MHz}$ elements each with a $70 \mathrm{~mm}$ focal distance. A 10-MHz diagnostic ultrasound imaging probe was positioned between the therapeutic elements and provides real-time images of the focal volume.

focal volume of the histotripsy transducer (Fig. 2). Good acoustic coupling was achieved by instilling a thin layer of degassed water between the shaved skin and plastic membrane.

Histotripsy pulses consisting of three cycle bursts of $1 \mathrm{MHz}$ acoustic energy at amplitudes exceeding $20 \mathrm{MPa}$ were delivered at a repetition frequency of $300 \mathrm{~Hz}$. During calibration, precise measurement of acoustic amplitude (in excess of $20 \mathrm{MPa}$ ) was confounded by formation of a bubble cloud around the hydrophone. In tumors not identifiable with US imaging, a $1 \mathrm{~cm}^{3}$ volume of tissue centered on the fiducial marker was targeted. Histotripsy treatment time averaged 5 minutes but varied in length based on subjective assessment of the quality of cavitation observed with real-time US feedback. In cases where cavitation was felt to be suboptimal, the acoustic amplitude was increased. Ten rabbits were eutha-

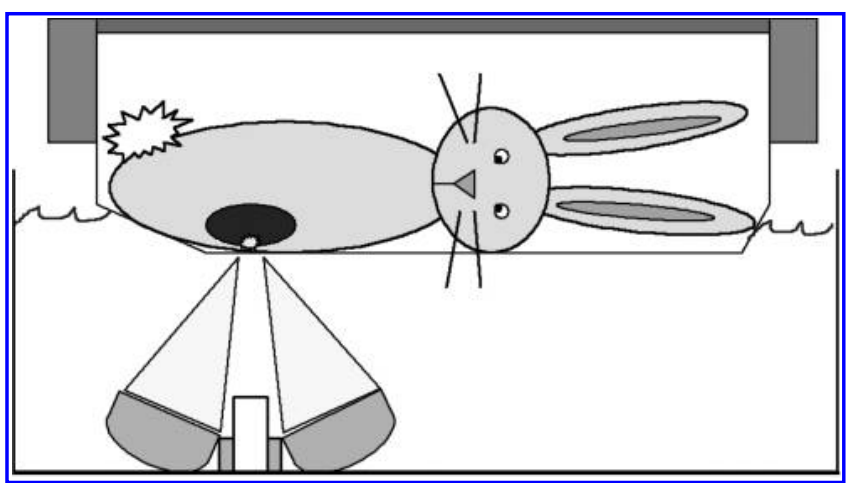

FIG. 2. Sketch of experimental setup. An anesthetized rabbit in flank position with the therapeutic transducer focal volume localized within the VX-2 tumor. 


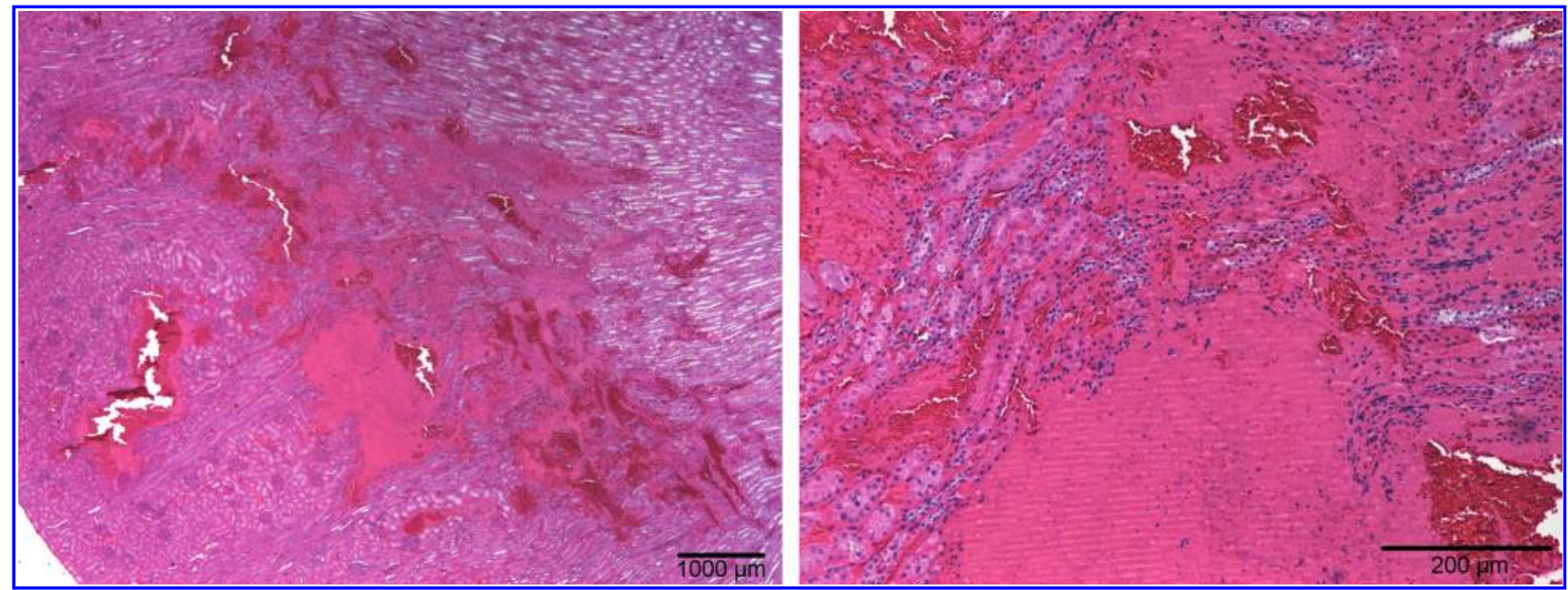

FIG. 3. H\&E-stained slide of normal rabbit kidney treated with histotripsy. Architectural damage is evident (left) with pockets of fractionated pink material within the treated region. At higher power (right), the pink material is seen to be largely acellular with small areas of localized hemorrhage. $\mathrm{H} \& \mathrm{E}=$ hematoxylin and eosin.

nized immediately after treatment. Five rabbits were recovered and monitored for 24 hours before euthanasia.

\section{Specimen analysis}

Kidneys and lungs were harvested at euthanasia, grossly inspected, and placed in formalin for 1 week. The kidneys were bi-valved, and tumor size was measured. The specimens were then processed for histologic examination. Hematoxylin and eosin slides of the kidneys were inspected for the presence of tumor and signs of cavitation effect. Metastatic foci were counted in one coronal whole-mount section of each left lung.

\section{Results}

Three normal rabbit kidneys (not implanted with tumor) were treated in vivo. In each case, a hyperechoic bubble cloud

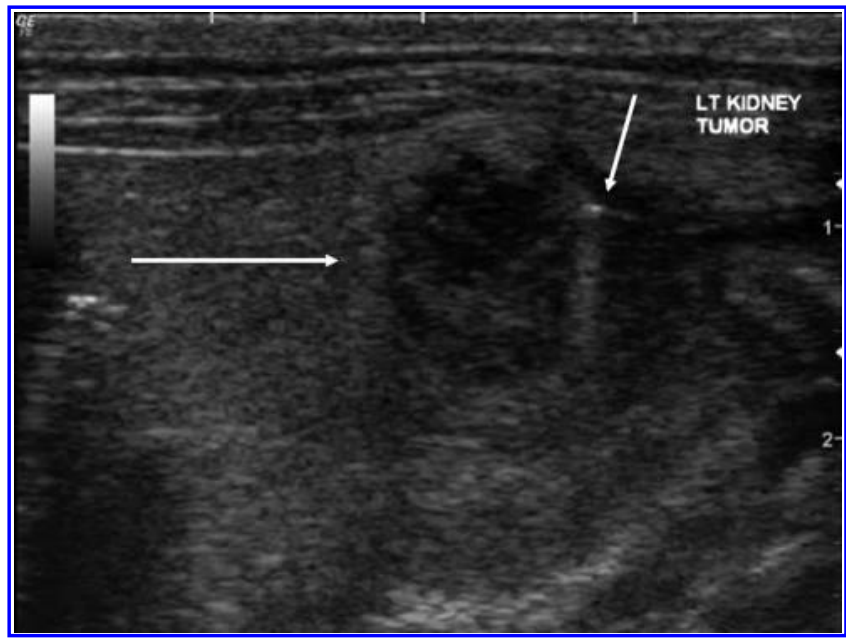

FIG. 4. A representative ultrasound image of VX-2 tumor within rabbit kidney after histotripsy treatment demonstrates a hypoechoic treatment defect. The left arrow represents the margin of tumor; right arrow points to surgical clip used as fiducial marker. (indicative of cavitation) was noted on real-time US imaging during histotripsy treatment. Small areas of hemorrhage within the targeted renal parenchyma and contained subcapsular hematoma were noted histologically. Regions of amorphous pink material admixed with particulate subcellular debris replaced the normal tissue architecture within the targeted volumes (Fig. 3).

Tumor was localized with US imaging in 24 of 27 implanted kidneys (Fig. 4). Average tumor diameter at harvest measured $7 \mathrm{~mm}$ (range 2-12) and demonstrated exophytic and endophytic growth patterns. Histology confirmed viable tumor in 27 of 27 implanted kidneys (20 treatment,

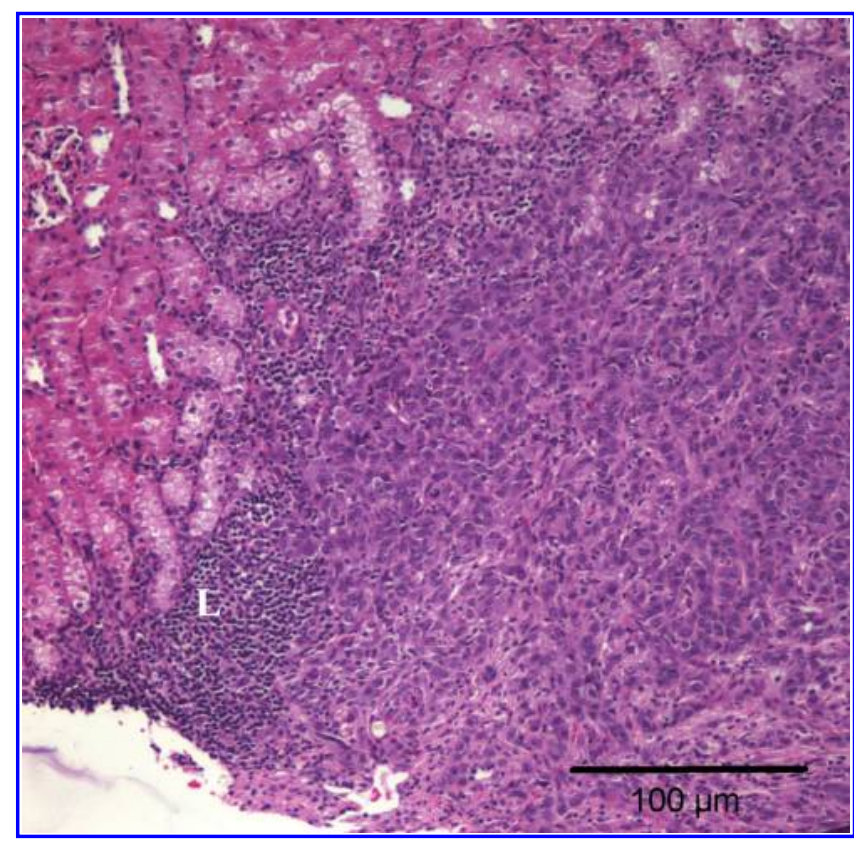

FIG. 5. H\&E-stained slide of untreated rabbit kidney with normal parenchyma (top left edge) and invasive VX-2 tumor staining strongly basophilic. A thin rim of leukocytic infiltration $(\mathrm{L})$ is noted at the tumor margin. 


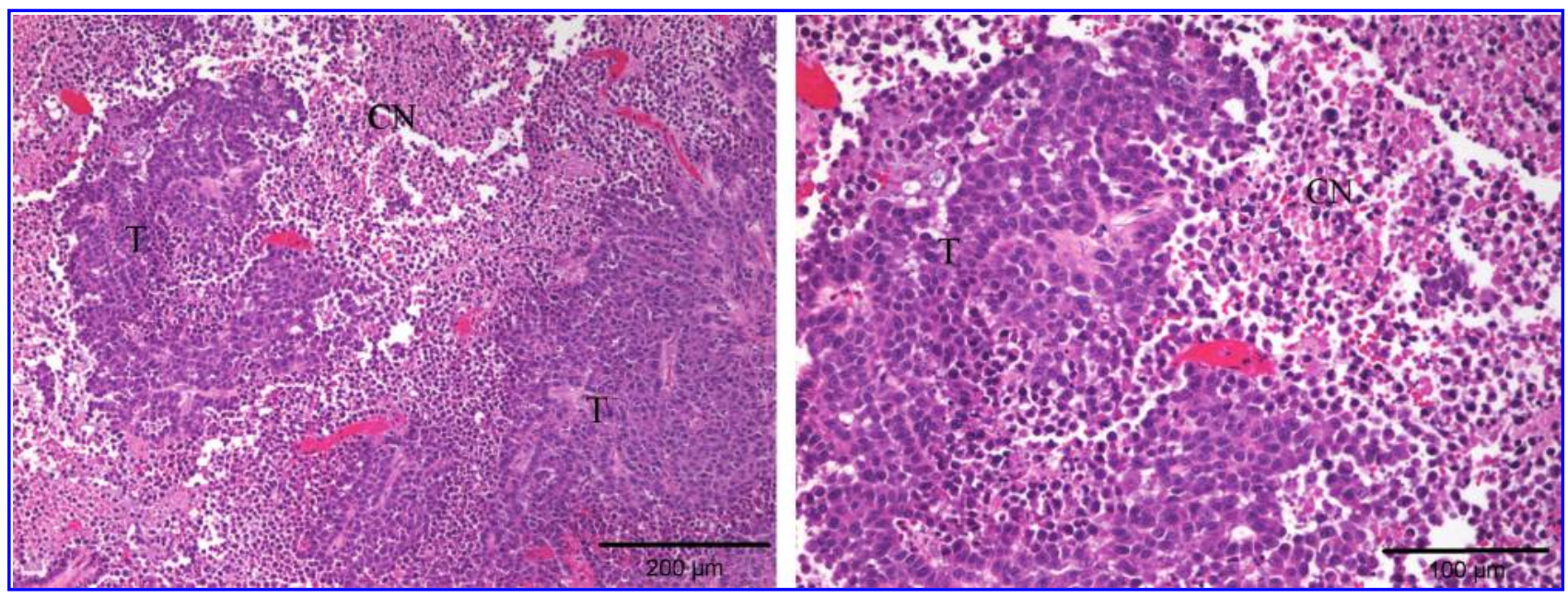

FIG. 6. H\&E-stained slide of untreated VX-2 tumor demonstrating central necrosis. Low-power (left) view reveals tumor (T) surrounded by zones of faint cellular staining representing central necrosis (CN). High-power view of the same region (right) reveals shrunken cell walls and absence of nuclei.

7 control). Tumor cells were identified by their irregular cell walls and strongly basophilic nuclei (Fig. 5). Zones of central necrosis characterized by faint cellular staining, shrunken cell walls, and absence of nuclei were identified in 14 of 27 tumors (Fig. 6). Infiltration of leukocytes was seen in a thin band at the interface between VX-2 tumor and normal renal parenchyma.

Histotripsy produced a bubble cloud visible with US imaging in all 20 targeted tumors. Gross inspection revealed small areas of hemorrhage within the targeted region of the tumor and subcapsular hematoma overlying the tumor (Fig. 7). In 16 of 20 treated tumors, acellular material and subcellular debris characteristic of histotripsy fractionation (Fig. 8) and distinct from central necrosis was clearly identified. Treated areas were bordered by intact basophilic tumor cells. Kidneys harvested 24 hours after treatment demonstrated similar areas of tissue fractionation; however, this treated region was nearly obscured by a predominantly neutrophilic inflammatory response (Fig. 9).
None of the rabbits suffered complication or death during VX-2 tumor implantation or histotripsy treatment, and none required early euthanasia. An average of 36 (range 3-51) metastatic tumor foci were counted on whole-mount lung sections from each tumor-implanted rabbit.

\section{Discussion}

The aggressiveness of VX-2 tumor proved advantageous in this study as $100 \%$ of implants were viable and 24 of 27 grew to a size identifiable with US imaging 2 weeks after implantation. As such, only three kidneys required histotripsy targeting based exclusively on the location of the fiducial marker. However, multiple lung metastases were found in all rabbits at harvest, which may be a limitation in future studies where longer follow-up is desirable.

Histotripsy ablates tissue by mechanical rather than thermal means and thus produces unique histologic findings. Cells within targeted volumes are fractionated, leaving regions de-

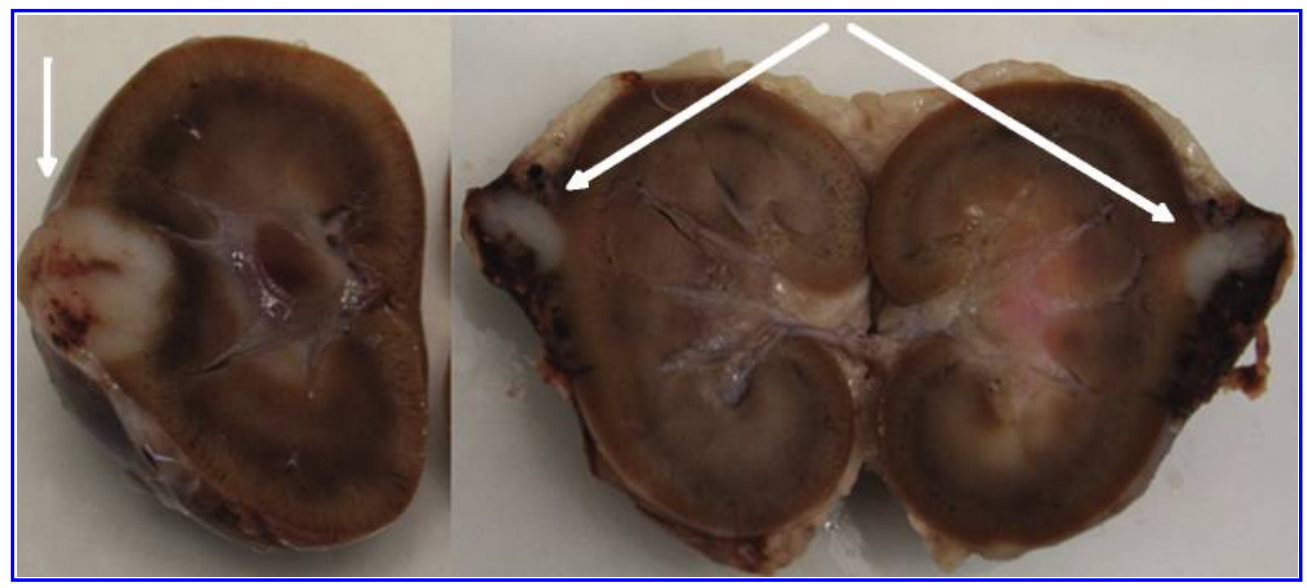

FIG. 7. Rabbit kidney as it appeared immediately after harvest. (Left) Large endo/exophytic VX-2 tumor is apparent as a white round area on surface of the bi-valved kidney (arrow). (Right) Bi-valved rabbit kidney demonstrating exophytic VX-2 tumor and small contained subcapsular hematoma resulting from histotripsy treatment (two arrows). 


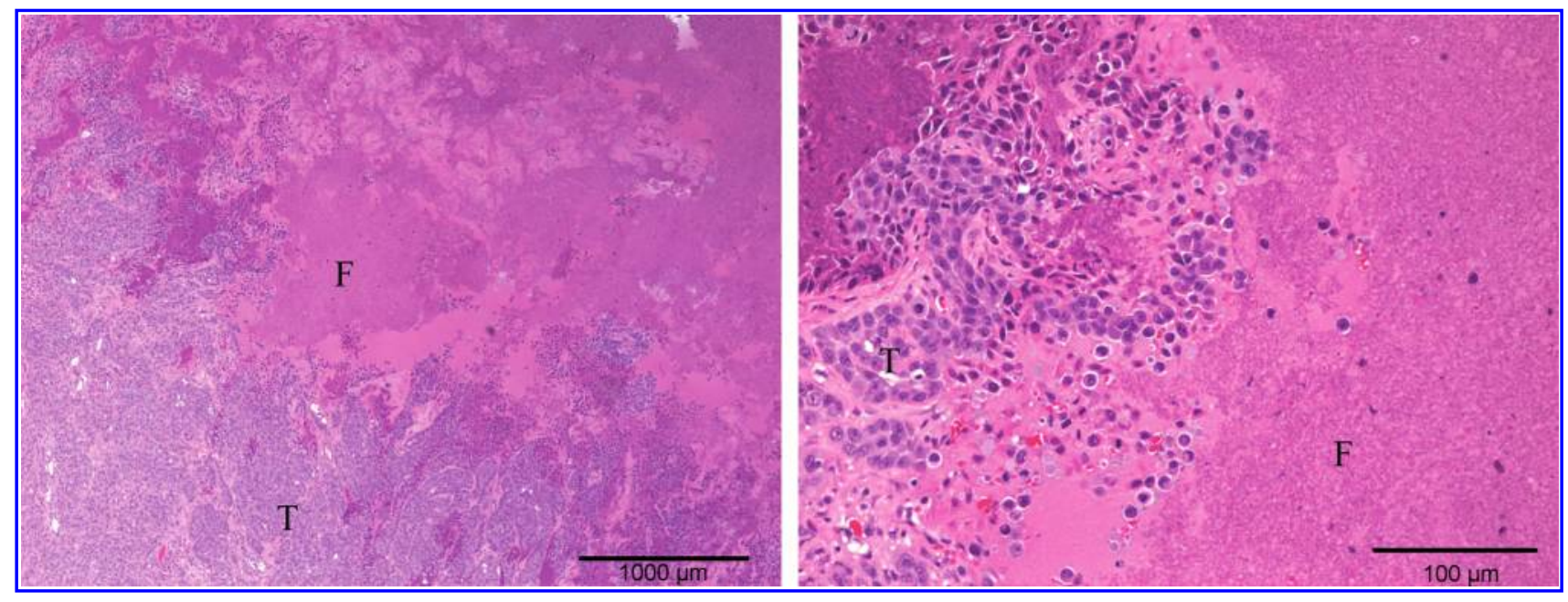

FIG. 8. H\&E-stained slide of renal implanted VX-2 tumor after treatment with histotripsy. (Left) Magnification of fractionated debris (F) consisting of pink predominantly acellular slurry of material adjacent to basophilic viable VX-2 tumor (T). (Right) Higher magnification confirms nests of VX-2 tumor adjacent to fractionated material composed of cytoplasmic and nuclear debris.

void of cellular and architectural features and containing only subcellular particulate debris amid homogenous amorphous material. ${ }^{1}$ This is in contradistinction to thermal coagulative necrosis produced by high-intensity focused US where cells are thermally fixed and though nonviable retain their architectural appearance. In the current study histotripsy applied to VX-2 tumor produced tissue fractionation and histologic patterns no different from those seen after histotripsy of normal renal tissues. ${ }^{1,5}$ These unique histologic patterns are easily distinguishable from those seen with central necrosis where faint cellular staining, shrunken cell walls, and absence of nuclei are typical findings of a tumor that outgrows its blood supply.

Histologic tissue fractionation was evident in $80 \%$ of treated tumors in this study, which confirms that histotripsy is capable of mechanical ablation of malignant tissue. No fractionation was seen in four tumors despite US confirmation of a bubble cloud. Three of these cases occurred in kidneys that were harvested 24 hours after treatment, and the inflammatory response may have obscured the zones of fractionation. Another possible explanation is that the method of bi-valving the kidney through the treated region during specimen preparation may have resulted in the region of interest being inadvertently discarded during processing. Typically, the front surface of the specimen block is shaved down to a completely flat surface before sections for slides are taken.

Histotripsy offers several potential advantages over conventional ablative modalities. Previous work in an in vitro porcine kidney model demonstrated that the collecting system was resistant to damage when exposed to histotripsy. In contrast, medullary tissue exhibited damage and cortical tissue was completely fractionated with identical histotripsy doses. ${ }^{14}$ This differential threshold for histotripsy tissue effect

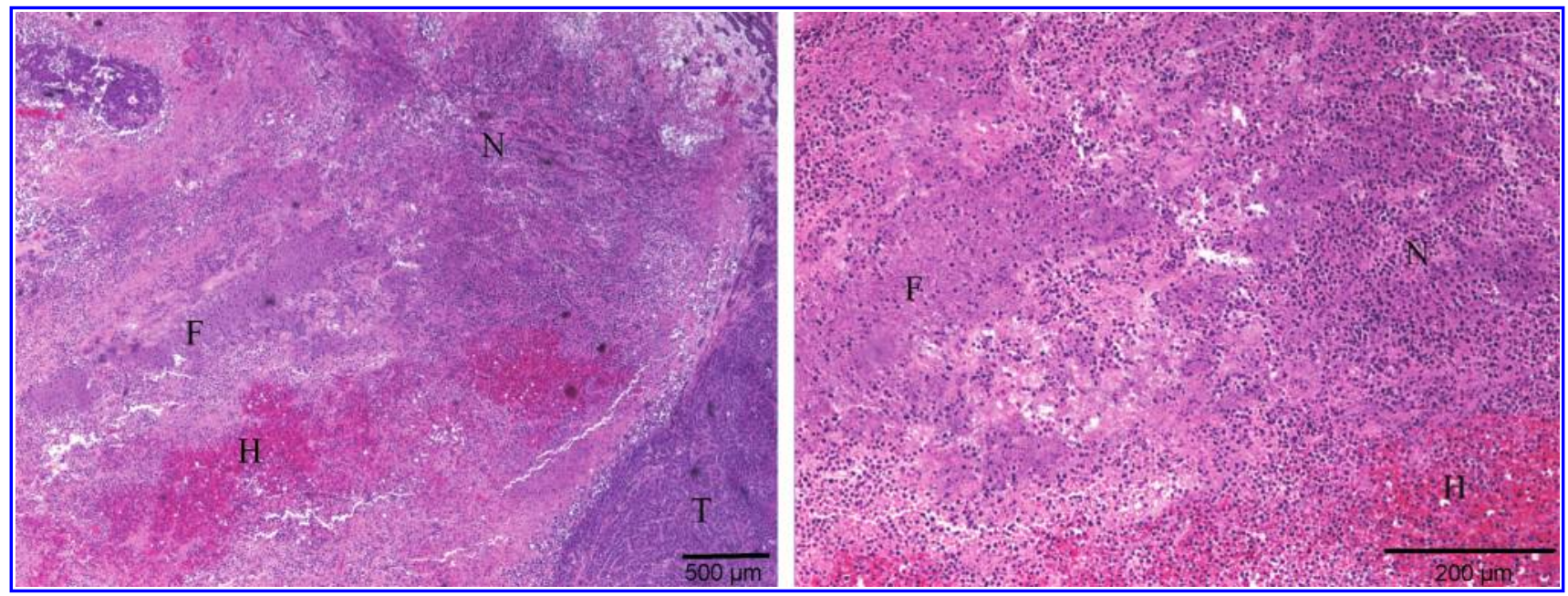

FIG. 9. H\&E-stained slide of implanted VX-2 tumor harvested 24 hours after histotripsy treatment. Low-power magnification (left) reveals areas of VX-2 tumor (T) with contained hemorrhage $(\mathrm{H})$ and acellular debris, consistent with histotripsy treatment $(\mathrm{F})$. Increased magnification (right) reveals a local predominantly neutrophilic inflammatory reaction surrounding the treated area $(\mathrm{N})$. 
is likely due to differences in tissue composition and may facilitate treatment of central renal lesions without damage to the collecting system. Further, the nonthermal mechanisms underlying histotripsy are not subject to the same limitations as conventional ablative modalities, where heat sink effects from adjacent large vessels can alter treatment boundaries and may preclude complete tumor destruction. ${ }^{15}$ A study in an in vivo rabbit model demonstrated that debris resulting from histotripsy fractionation of normal kidney tissue was completely resorbed within 60 days of treatment. ${ }^{5}$ Should this finding be validated with ablation of larger lesions, it could simplify postablation surveillance strategies that are complicated by the slow and incomplete resorption of many cryoablated and thermally coagulated tumors after tumor treatment. ${ }^{16}$ Experimental evidence from a canine prostate model suggests that histotripsy, in addition to being noninvasive, may also exhibit hemostatic properties that could further lower the risk of hemorrhage during tumor ablation. ${ }^{17}$

One particularly useful advantage of histotripsy is the immediate feedback achieved with US observation of the cavitation bubble cloud. This method of feedback provided confirmation of cavitation in all targeted kidneys and allowed subjective monitoring of the quality of cavitation. In four cases, the quality of cavitation was weak, when only a small or intermittent bubble cloud was seen, or when formation of the bubble cloud was delayed. Suboptimal cavitation can be attributed to a number of factors, including poor acoustic coupling, attenuation from a long skin to tumor distance, and aberration due to ribs or an overlying skin incision in the acoustic path.

The initial results achieved in a VX-2 tumor model are encouraging and demonstrate the feasibility of histotripsy treatment of malignant tissue. However, the translation of these findings to other tumor models is uncertain and requires further investigation. Additional work is also planned to better understand the systemic effects that may result from histotripsy on malignant tissues.

\section{Conclusions}

Transcutaneous application of histotripsy to implanted VX2 tumor in rabbit kidney produced fractionation of malignant tissue. These findings support the further study and development of histotripsy for potential oncologic application.

\section{Disclosure Statement}

Timothy L. Hall and William W. Roberts are inventors of intellectual property licensed to HistoSonics Inc., hold stock in HistoSonics Inc., and consult for HistoSonics Inc.

\section{References}

1. Roberts WW, Hall TL, Ives K, et al. Pulsed cavitational ultrasound: A noninvasive technology for controlled tissue ablation (Histotripsy) in the rabbit kidney. J Urol 2006;175: 734-738.

2. Xu Z, Raghavan M, Hall TL, et al. High speed imaging of bubble clouds generated in pulsed ultrasound cavitational therapy-histotripsy. IEEE Trans Ultrasoun Ferroelectr Freq Control 2007;54:2091-2101.

3. Parsons JE, Cain CA, Fowlkes JB. Characterizing pulsed ultrasound therapy of production of cavitationally-induced lesions. Proceedings of the 4th International Symposium on Therapeutic Ultrasound, 2004.

4. Xu Z, Ludomirsky A, Eun LY, Hall TL, et al. Controlled ultrasound tissue erosion. IEEE Trans UFFC 2004;51: 726-736.

5. Hall TL, Kieran K, Ives K, et al. Histotripsy of rabbit renal tissue in vivo: Temporal histologic trends. J Endourol 2007; 21:1159-1165.

6. Mabjeesh NJ, Avidor Y, Matzkin H. Emerging nephron sparing treatments for kidney tumors: A continuum of modalities from energy ablation to laparoscopic partial nephrectomy. J Urol 2004;171:553-560.

7. Kohrmann KU, Michel MS, Gaa J, et al. High intensity focused ultrasound as noninvasive therapy for multilocal renal cell carcinoma: Case study and review of the literature. J Urol 2002;167:2397-2403.

8. Kieran K, Hall TL, Parsons JE, et al. Refining histotripsy: Defining the parameter space for the creation of nonthermal lesions with high intensity, pulsed focused ultrasound of the in vitro kidney. J Urol 2007;178:672-676.

9. Miao Y, Ni Y, Bosmans H, et al. Radiofrequency ablation for eradication of renal tumors in a rabbit model by using a cooled-tip electrode technique. Ann Surg Oncol 2001;8: 651-657.

10. Zhou C, Fi F, Qin Y, et al. Non-thermal ablation of rabbit liver VX2 tumor by pulsed high intensity focused ultrasound with ultrasound contrast agent: Pathologic characteristics. World J Gastroenterol 2008;14:6743-6747.

11. Miao $Y$, Ni Y, Mulier S, et al. Treatment of VX2 liver tumor in rabbits with "Wet" electrode mediated radio-frequency ablation. Eur Radiol 2000;10:188-194.

12. Lian X, Tang G, Cheng Y, Zhou B. Evaluation of rabbit rectal VX2 carcinoma model using computed tompography and magnetic resonance imaging. World J Gastroenterol 2009; 15:2139-2144.

13. Ho $Y$, Huang $Y$, Lin $C$, et al. Application of radiofrequency ablation of renal VX2 tumors by cooled-tip electrode in a rabbit model. J Endourol 2009;23:677-684.

14. Lake AM, Xu Z, Wilkinson JE, et al. Renal ablation by histotripsy-does it spare the collecting system? J Urol 2008; 179:1150-1154.

15. Gervais DA, McGovern FJ, Wood BJ, et al. Radio-frequency ablation of renal cell carcinoma: Early clinical experience. Radiology 2000;217:665-672.

16. Hegarty NJ, Gill IS, Desai MM, et al. Probe-ablative nephron-sparing surgery: Cryoablation versus radiofrequency ablation. Urology 2006;68 supp 1A:7-13.

17. Wheat JC, Hall TL, Hempel RC, et al. Prostate histotripsy in an anticoagulated model. Urology 2009 2010;75:207-211.

Address correspondence to: William W. Roberts, M.D.

Department of Urology University of Michigan

1500 E. Medical Center Drive

Taubman Center, Room 3879

Ann Arbor, MI 48109-5330

E-mail: willrobe@umich.edu

\section{Abbreviations Used}

$\mathrm{H} \& \mathrm{E}=$ hematoxylin and eosin $\mathrm{US}=$ ultrasound 